\title{
Defining airflow limitation and chronic obstructive pulmonary disease: the role of outcome studies
}

\author{
Bruce Culver
}

Affiliation: Division of Pulmonary and Critical Care Medicine, Dept of Medicine, University of Washington, Seattle, WA, USA.

Correspondence: Bruce Culver, Division of Pulmonary and Critical Care Medicine, University of Washington B0X 356522, Seattle WA, 98195-6552, USA. E-mail: bculverQuw.edu

0

@ERSpublications

Outcome studies in high risk as well as general populations are needed to improve the recognition of early COPD http://ow.ly/MkxvJ

A guideline to the interpretation of pulmonary function tests, published 20 years ago, made a distinction between the related tasks of "(1) the classification of the derived values with respect to a reference population... and (2) the integration of the spirometric values into the diagnosis, therapy and prognosis for an individual patient." [1]. The first of these is very straightforward, when appropriate reference data are available, but the latter is difficult in the early stages of disease and will have a degree of uncertainty for any individual. Confusion arises when the distinction between these two aspects of interpretation is blurred.

It is widely accepted that the primary index of airflow limitation (or airway obstruction) is the ratio of forced expiratory volume in $1 \mathrm{~s}$ (FEV1) to forced vital capacity (FVC), the $\mathrm{FEV}_{1} / \mathrm{FVC}$ (or $\mathrm{FEV}_{1} / \mathrm{VC}$ ) [2]. In healthy normal nonsmokers, this ratio decreases progressively with age, with female values slightly higher than male. It is also widely accepted that the normal range of physiological parameters is defined in a way that will encompass $95 \%$ of a normal, healthy population. For spirometric parameters, where only low values are considered abnormal, this is achieved by setting the lower limit of normal (LLN) at the 5th percentile of a reference population of the same age, height, sex, and ethnicity. When the population distribution above and below the median is not skewed, this is equivalent to $-1.64 \mathrm{sD}$. Reference data providing a statistically determined 5th percentile LLN for $\mathrm{FEV}_{1} / \mathrm{FVC}$ have been available, at least for a Caucasian population, for $>30$ years [3], with newer studies offering larger, more inclusive, populations and more sophisticated statistical analyses [4-6].

In 2001, in an effort to increase awareness and recognition of chronic obstructive pulmonary disease (COPD) particularly in less developed areas of the world, the Global Initiative for Chronic Obstructive Lung Disease (GOLD) group published a definition of COPD that included airflow limitation shown by a post-bronchodilator FEV1/FVC of $<70 \%$ (0.70) [7]. The LLN of this ratio, decreasing with age, crosses 0.70 at $~ 46$ years in males and 54 years in females [5], so this fixed cut-off does provide an approximation of the LLN in the mid-range of age, where screening or case-finding for COPD is most likely to be helpful. However, it has commonly been applied throughout the age spectrum and the adoption of this fixed ratio definition by other COPD guidelines [8-10] has, unfortunately, led some clinicians to inappropriately consider this to be a generally applicable definition of airflow limitation. It is very clear, and acknowledged in subsequent GOLD updates [11], that this definition could lead to under-recognition of obstructive disease in young individuals and over-diagnosis in older individuals. Because normal FEV1/FVC values are higher in women the fixed cut-off also creates a sex bias, with young females more likely to be under-recognised and older males more likely to be over-diagnosed. The latter was soon demonstrated in a 
study from Bergen in Norway, where one third of healthy, never-smoking males met this flawed criterion for COPD [12] and this misclassification has been documented in many subsequent reports [13-19], mostly focussing on the 60-80-year age range.

The paper by TURKeshi et al. [20], in the current issue of the European Respiratory Journal, takes advantage of the extended age range and age-specific variance incorporated in the recent Global Lungs Initiative (GLI) reference data [6] in order to examine this question in elderly adults. In a population based sample of adults aged $>80$ years, $9 \%$ fell below the 5th percentile LLN of the GLI 2012 predictions (GLI-LLN) while an additional $18 \%$ met the $<0.70$ criterion. Thus, of those with a ratio $<0.70$, two out of three fell within the expected range of a normal population. The relatively low prevalence of airflow limitation by GLI-LLN reflects the female preponderance in this age range; this criterion was met by $19 \%$ of males but only $3 \%$ of females. The importance of spirometry in the diagnosis of COPD is emphasised by the observation that less than half of the individuals who were identified, by their general practitioners, as having COPD had airflow limitation confirmed by an FEV1/FVC below the LLN; the correlation with the $<0.70$ criterion was also poor. Using the measures of all-cause mortality and time to the first, unplanned, hospitalisation, the authors examined the outcomes of those with an FEV1/FVC below the GLI-LLN (all were also $<0.70$ in this age range) versus those $<0.70$ but $>$ GLI-LLN, the discordant group. Only airflow limitation identified by GLI-LLN was independently associated with mortality, with an adjusted hazard ratio of 2.10, while the discordant group identified only by $\mathrm{FEV} 1 / \mathrm{FVC}<0.70$ had no significantly higher risk for mortality or hospitalisation. This is an important observation because it has been suggested that, rather than over-diagnosis, the $<0.70$ cut-off might provide a useful increase in sensitivity [21, 22]. However, it should be recognised that any increase in cases found by the fixed ratio would occur only in older individuals, progressively with age and predominantly in males, whereas the desired result would be age and gender neutral and applicable at ages 45-60 years, where the potential benefit is greatest. The Turkeshi et al. [20] study shows that in this elderly, population based sample, any increase in the detection of early disease by the $<0.70$ cut-off is outweighed by the misclassification of a larger number of healthy seniors.

The goal of early identification of patients progressing toward clinically important COPD is laudable and should be approachable with further attention to the outcomes of well-described relevant populations. The seminal long-term study by FLETCHER and PETO [23] showed that the development of airflow limitation in COPD was characterised by a slow progressive fall in FEV1, averaging only about twice the expected decline of normal aging. This, in combination with the wide normal-range of spirometry values and the relative insensitivity of forced airflow measurements to increases in small airway resistance, means that early stages of disease will inevitably be present before the FEV1/FVC falls below the population LLN, especially for an individual who starts with a high normal value. This was demonstrated when CAsIo et al. [24] performed a battery of lung function tests on individuals scheduled for resection of small lung lesions, then analysed lung parenchymal tissue, remote from the lesion, for evidence of small airway disease. As the pathology score increased from grade 1 , minimal abnormalities, to grade 4 there was a stepwise decline in mean FEV1/FVC, but it did not become significantly different from the control group until grade 3 pathology was present. Of note, none of the five tests of the small-airway dysfunction of interest, at that time, outperformed the FEV1/FVC ratio. With more recent technology, the presence of emphysema has been documented by computed tomography while spirometry remains within normal limits [25].

The difficulty in confirming suspected COPD at an early stage invites consideration of using a more sensitive criterion for airflow limitation in higher risk individuals, analogous to the stepped cut-offs for tuberculin skin testing: $5 \mathrm{~mm}, 10 \mathrm{~mm}$, or $15 \mathrm{~mm}$ for high, moderate and low-risk individuals, respectively. A strategy, more physiologically and statistically rational than a fixed ratio, would be to raise the percentile LLN to 7.5 or 10 in high-risk individuals. VAZ Frogoso, et al. [26] examined this possibility by comparing the outcomes of individuals with $\mathrm{FEV} 1 / \mathrm{FVC}<5$ th percentile to those at progressively higher percentile strata, 5th-10th, 10th-15th, etc. with those $>25$ th percentile serving as the reference population. As expected those <5th percentile LLN had increased subsequent all-cause mortality, while there was no indication of this for those $>10$ th percentile. The borderline strata between the 5 th-10th percentiles had a significantly elevated unadjusted odds ratio of 1.59 for mortality, but when adjusted for risks, including smoking, this fell to 1.26 and the confidence interval just crossed 1 . Thus, the independent effect of this degree of airflow limitation was not shown to be significant, but for assessing an individual's probability of disease and prognosis the combined risks should be more relevant. Any increase in diagnostic sensitivity gained from adjusting the LLN upward is inevitably accompanied by a loss of specificity so, to minimise false positives, it is essential to evaluate any risk stratified cut points with outcomes in appropriate populations, including the high risk individuals most likely to be referred for lung function testing.

The Turkeshi et al. [20] study in this issue, the Vaz Frogoso et al. [26] study, noted earlier, and most of the currently available outcome studies have been based upon general populations with a relatively low 
prevalence of airway disease. The situation facing a clinical pulmonary function laboratory or an individual practitioner is quite different. When spirometry is done on patients with symptoms or relevant risk factors the prior probability of airway disease is greatly increased and spirometry values clearly above the healthy population LLN still may, more likely than not, be associated with early airway disease. We can readily and accurately classify spirometry values with respect to a reference population and the recent availability of the GLI-2012 data provides an opportunity for commonality across regions [27]. However, to integrate these values into the diagnosis, therapy and prognosis for an individual patient, we need to move beyond the false dichotomy of normal/abnormal. We need to develop the risk profiles and outcome data that would allow us to make a rational statement about the probability of disease at any point on the spectrum of FEV1/FVC from mid-normal down to the LLN and below.

\section{References}

1 Standardization of spirometry, 1994 update: American Thoracic Society. Am J Respir Crist Care Med 1995; 152: 1107-1136.

2 Pellegrino R, Veii G, Brusasco V, et al. Interpretative strategies for lung function tests. Eur Respir J 2005; 26 : 948-968.

3 Crapo RO, Morris AH, Gardner RM. Reference spirometric values using techniques and equipment that meet ATS recommendations. Am Rev Respir Dis 1981; 123: 659-664.

4 Hankinson JL, Odencrantz JR, Fedan KB. Spirometric reference values from a sample of the general US population. Am J Respir Crist Care Med 1999; 159: 179-187.

5 Stanojevic S, Wade A, Stocks J, et al. Reference ranges for spirometry across all ages: a new approach. Am J Respir Crist Care Med 2008; 177: 253-260.

6 Quanjer PH, Stanojevic S, Cole TJ, et al. Multi-ethnic reference values for spirometry for the 3-95-year age range: the global lung function 2012 equations. Eur Respir J 2012; 40: 1324-1343.

7 Pauwels RA, Buist AS, Calverley PMA, et al. Global strategy for the diagnosis, management, and prevention of chronic obstructive pulmonary disease: NHLBI/WHO Global Initiative for Chronic Obstructive Lung Disease (GOLD) Workshop summary. Am J Respir Crist Care Med 2001; 163: 1256-76.

8 Celli BR, MacNee W, Agusti A, et al. Standards for the diagnosis and treatment of patients with COPD: a summary of the ATS/ERS position paper. Eur Respir J 2004; 23: 932-946.

9 Qaseem A, Wilt TJ, Weinberger SE, et al. Diagnosis and management of stable chronic obstructive pulmonary disease: a clinical practice guideline update from the American College of Physicians, American College of Chest Physicians, American Thoracic Society, and European Respiratory Society. Ann Intern Med 2011; 155: 179-191.

10 National institute for Health and Clinical Excellence. Chronic obstructive pulmonary disease: management of chronic obstructive pulmonary disease in adults in primary and secondary care (partial update). www.nice.org.uk/ guidance/cg101. Date last accessed: March 10, 2015. Date last updated: July, 2014.

11 Rabe KF, Hurd S, Anzueto A, et al. Global strategy for the diagnosis, management, and prevention of chronic obstructive pulmonary disease: GOLD executive summary. Am J Respir Crist Care Med 2007; 176: 532-555.

12 Hardie JA, Buist AS, Vollmer WM, et al. Risk of over-diagnosis of COPD in asymptomatic elderly never-smokers. Eur Respir J 2002; 20: 1117-1122.

13 Celli BR, Halbert RJ, Isonaka S, et al. Population impact of different definitions of airway obstruction. Eur Respir J 2003; 22: 268-273.

14 Shirtcliffe P, Weatherall M, Marsh S, et al. COPD prevalence in a random population survey: a matter of definition. Eur Respir J 2007; 30: 232-239.

15 Schermer TRJ, Smeele IJM, Thoonen BPA, et al. Current clinical guideline definitions of airflow obstruction and COPD overdiagnosis in primary care. Eur Respir J 2008; 32: 945-952.

16 Swanney MP, Ruppel G, Enright PL, et al. Using the lower limit of normal for the FEV $/$ FVC ratio reduces the misclassification of airway obstruction. Thorax 2008; 63: 1046-1051.

17 Miller MR, Quanjer PH, Swanney MP, et al. Interpreting lung function data using $80 \%$ predicted and fixed thresholds misclassifies more than $20 \%$ of patients. Chest 2011; 139: 52-59.

18 Mohamed Hoesein FA, Zanen P, Lammers JW, et al. Lower limit of normal or FEV $1 / \mathrm{FVC}<0.70$ in diagnosing COPD: an evidence-based review. Respir Med 2011; 105: 907-915.

19 van Dijk WD, Gupta N, Tan WC, et al. Clinical relevance of diagnosing COPD by fixed ratio or lower limit of normal: a systematic review. COPD 2014; 11: 113-120.

20 Turkeshi E, Vaes B, Andreeva E, et al. Airflow limitation by the Global Lungs Initiative equations in a cohort of very old adults. Eur Respir J 2015; 46: 123-132.

21 Mannino DM, Buist AS, Vollmer WM. Chronic obstructive pulmonary disease in the older adult: what defines abnormal lung function? Thorax 2007; 62: 237-241.

22 Mannino DM, Diaz-Guzman E. Interpreting lung function data using 80\% predicted and fixed thresholds identifies patients at increased risk of mortality. Chest 2012; 141: 73-80.

23 Fletcher C, Peto R. The natural history of chronic airflow obstruction. Br Med J 1977; 1: 1645-1648.

24 Casio M, Ghezzo H, Hogg JC, et al. The relations between structural changes in small airways and pulmonary-function tests. N Engl J Med 1978; 298: 1277-1281.

25 Oelsner EC, Hoffman EA, Folsom AR, et al. Association between emphysema-like lung on cardiac computed tomography and mortality in persons without airflow obstruction: a cohort study. Ann Intern Med 2014; 161: 863-873.

26 Vaz Fragoso CA, Concato J, McAvay G, et al. The ratio of FEV1 to FVC as a basis for establishing chronic obstructive pulmonary disease. Am J Respir Crist Care Med 2010; 181: 446-451.

27 Swanney MP, Miller M. Adopting universal lung function reference equations. Eur Respir J 2013; 42: 901-903. 